\title{
"Te extraño, te olvido, te amo de nuevo". La construcción del amor en la literatura juvenil argentina
}

\author{
Por: Licda. Giulana A. Pates ${ }^{1}$, Universidad de La Plata, Argentina
}

Recibido: 14 de febrero, 2018

Aceptado: 19 de abril, 2018

\section{RESUMEN}

Recociendo que para analizar objetos literarios es necesario desbordar los límites del texto, este artículo apunta a indagar en las mediaciones existentes entre la literatura y el entramado social, es decir, los rasgos específicos del texto literario y la red de relaciones de la que es parte. En particular, se centrará en la literatura juvenil romántica argentina para preguntarse cómo está configurado este campo, qué matrices discursivas en torno del amor se construyen y cómo es leída y apropiada por parte del público lector juvenil. Para ello se analizarán los libros S.O.S. Tengo mi primera cita (Dritsos, 2014) y Diario de una ruptura (Dritsos, 2016).

En primera instancia, se contextualizarán los libros seleccionados dentro del mercado editorial argentino y se recuperará la trayectoria de su autora para dar cuenta de sus condiciones sociales de producción. En segundo lugar, se desglosará el análisis de ambos libros en torno a los modos en que se construye el amor a partir de dos matrices discursivas: el amor romántico y el amor contingente. Finalmente, se reconstruirán los espacios por donde han circulado estos libros, así como también los modos en que han sido leídos y apropiados en Argentina.

\section{ABSTRACT}

\section{"I miss you, I forget you, I love you again." The construction of love in Argentinian young adult literature}

When we analyze literary objects, it is necessary to go beyond the limits of the text. This article aims to look at the mediations between the literary and social frameworks; that is to say, the specific features of the literary text and the relationship network to which it belongs. We shall focus specifically on Argentinian romantic young adult literature to reflect on how the literary field is built, what discursive matrices on love are constructed, and how it is read by young readers. To do so, Georgina Dritsos' books S.O.S. Tengo mi primera cita (2014) and Diario de una ruptura (2016) shall be analyzed.

First of all, these two books shall be set within to the Argentinian editorial market as well as the author's literary career will be mentioned. Secondly, the ways in which love is constructed in both books will be analyzed following two discursive matrices: romantic love and contingent love. Finally, the places where the books have circulated and the ways they have been read in Argentina it shall be summarized.

1 Giulana Pates es licenciada en Comunicación Social con orientación en Planificación Comunicacional, graduada de la Universidad Nacional de la Plata, Argentina. Actualmente realiza su tesis para optar por la maestría en Sociología de la Cultura y Análisis Cultural, en la Universidad Nacional de San Martín, Argentina. Contacto: giulianapates@hotmail.com. 
"Te extraño, te olvido, te amo de nuevo". La construcción del amor en la literatura juvenil argentina

Construir objetos de estudio literarios no implica que preguntarse únicamente por el modo en que están escritos los textos o la biografía de su autor. Si bien pueden constituirse en dimensiones a indagar, un abordaje que pretenda no ser parcial invita a asumir una mirada que exceda el análisis textualista. En este sentido, se entiende que el objeto de estudio tiene que ser el hecho literario en tanto hecho social, es decir, inscripto en relaciones sociales y el marco de una sociedad y una cultura específicas, en donde participan sujetos e instituciones. No hay una separación dicotómica entre un "adentro" literario, textual y un "afuera" social, extratextual. Se debe atender las relaciones que se entablan entre estos dos espacios que no son externos entre sí, sino que se implican mutuamente.

Para ello, la perspectiva de la sociología de la literatura permite entender la significación de una obra más allá de las intenciones de su autor o su construcción interna. Articular las herramientas de la sociología y la literatura ayuda a acercarse a una constelación de productos que se reúnen bajo la categoría de literatura e inscribirla en un contexto de producción y circulación más amplios. El desafío, permanente y dialógico, transformado en abordaje metodológico, consiste en atender el universo delimitado por el texto -sus rasgos de constitución específicos- y la red de relaciones que lo insertan en el entramado social.

Siguiendo esta línea propuesta por la sociología de la literatura, se señalan tres ámbitos de indagación en donde se focalizará el artículo para preguntarse por las mediaciones entre las obras literarias y los contextos socio-históricos de los que son parte. Por un lado, las condiciones sociales de producción de las obras, entendidas como las relaciones de la literatura con otros campos sociales, así como también la organización del campo literario -sus instituciones, las posiciones que ocupan los autores, sus trayectorias y vínculos de sociabilidad, y las formaciones culturales-. En segundo lugar, la puesta en forma de los textos literarios, es decir, las maneras de hacer literarias, las elecciones formales y genéricas dentro del "espacio de posibles" (Bourdieu, 1992) de una época y lugar dados que refractan el mundo social. Por último, las condiciones de circulación y apropiación de las obras literarias, así como sus usos dentro o fuera del campo literario (Sapiro, 2016).

Desde este posicionamiento teórico y metodológico -entonces- y como parte de una investigación más amplia en el marco de la tesis doctoral de quien escribe este artículo, en torno a la literatura juvenil romántica², se abordará aquí el caso de dos libros juveniles argentinos de reciente publicación: S.O.S. Tengo mi primera cita (2014) y Diario de una ruptura (2016), ambos escritos por Georgina Dritsos, para preguntarse por esa red de relaciones formada entre estos textos y el entramado social. En este sentido, no es este un análisis lingüístico que ahonde en las formas de la escritura de los libros ni se hará una evaluación de su calidad estética. Se plantea, en cambio, poner en relación estas producciones literarias con su contexto sociocultural y las posibilidades históricas que permitieron que se escribieran y editaran.

Por tanto, este artículo partirá de presentar y describir los modos de producción de la literatura juvenil en Argentina, centrándose en la editorial que ha publicado los dos libros seleccionados y la inscripción de su autora en el campo literario. A continuación, se analizarán estas producciones con la intención de indagar los sentidos sociales en torno del amor que ellas refractan a partir de las elecciones genéricas y formales realizadas. Finalmente, se planteará un acercamiento a sus condiciones de circulación y apropiación en el campo literario.

\section{COORDENADAS DE LA LITERATURA JUVENIL ARGENTINA}

Poner la mirada en las condiciones sociales de producción de las obras literarias obliga a prestar atención a la situación de la literatura, en este caso juvenil, respecto de otros espacios sociales, así como

2 Desde el 2017, la autora cuenta con una beca doctoral del Consejo Nacional de Investigaciones Científicas y Técnicas (CONICET), cuyo título es "Por ese palpitar. Las experiencias de lectura de literatura romántica de circulación masiva como nueva adscripción identitaria juvenil", bajo la dirección de la Dra. Florencia Saintout y cuyo lugar de trabajo es el Instituto de Estudios Comunicacionales en Medios, Cultura y Poder "Aníbal Ford". A parti de ella, se propone analizar las matrices culturales del amor que se construyen en las novelas juveniles románticas contemporáneas, así como también indagar en las experiencias de lectura de estas por parte de jóvenes de sectores medios urbanos de la Ciudad Autónoma de Buenos Aires (CABA) y La Plata, en Argentina. 
también su organización interna. La literatura como espacio de producción cultural se ha autonomizado a partir de la profesionalización de los escritores, la creación de instituciones específicas que la regulan y marcan sus límites (desde la escuela y la universidad que la construyen como un saber académico hasta las organizaciones y academias profesionales, los eventos como ferias y festivales hasta la otorgación de premios), la formación de un cuerpo de especialistas y la constitución de un mercado propio.

La literatura juvenil, en particular, es motivo de reflexión en el campo pedagógico, porque parte de su circulación se da dentro del sistema escolar, es objeto de estudio de investigaciones académicas y es uno de los géneros más rentables para el mercado editorial local. Respecto de este último punto, partiendo de los datos que proporcionan los informes y las estadísticas anuales construidas por la Cámara Argentina del Libro, se sostiene que la publicación de Literatura Infantil y Juvenil (LIJ) ha aumentado en cantidad de títulos en los últimos años, posicionándose como uno de los tres sectores más editados en el país. En los últimos ocho años, ha representado entre $15 \%$ y $18 \%$ del total de libros publicados y registrados en la Argentina, mientras que en el Sector Editorial Comercial (SEC) alcanzó 22\% en el primer semestre del 2017. En los informes, las editoriales son clasificadas en tres sectores: Sector Editorial Comercial, Autoedición y Otros, en donde se incluyen microemprendimientos, instituciones educativas y entidades públicas. Cabe destacar, además, que la literatura (narrativa y poesía) representa entre $20 \%$ y $25 \%$, y las Ciencias Sociales/Humanas, entre $20 \%$ y $24 \%$, de acuerdo con los datos proporcionados en los informes anuales de producción del libro argentino.

Si bien la literatura juvenil tiene una cantidad menor de libros publicados que los de literatura infantil -se los considera dentro de una misma categoría en estos informes-, el número de literatura juvenil en circulación aumenta si se incorporan al análisis las publicaciones extranjeras, sobre todo las norteamericanas, inglesas y españolas, que son las que más se venden en este sector. Así, se puede ver que el $16 \%$ de los libros más vendidos durante el año 2014 pertenecieron a la LIJ, y el 86\% de esa porción fueron novelas juveniles (Cámara Argentina de Publicaciones, 2015).

En este marco de producción editorial es que se ubican los dos libros que se proponen analizar: S.O.S. Tengo mi primera cita (2014) y Diario de una ruptura (2016), ambos escritos por la escritora, periodista y Community Manager Georgina Dritsos, y editados por V\&R Editoras. Esta editorial se fundó en 1996 en Argentina y en los últimos años, abrió sucursales en México y Brasil, lo que le posibilitó ampliar la distribución de sus libros en el resto de América Latina, así como también en Estados Unidos y España. Forma parte del Sector Editorial Comercial (SEC), que representa el $42 \%$ de las ediciones en Argentina, dentro del cual la concentración por cantidad de ejemplares y novedades editadas se reduce principalmente a seis editoriales: Planeta, PenguinRandomHouse, Grupo Prisa, Holtzbrinck, Longseller y Thompson Reuters (Cámara Argentina de Publicaciones, 2015).

V\&R Editoras, en particular, se caracteriza por editar publicaciones infantiles y juveniles, desde libros para colorear hasta novelas, asimismo, por tener en su catálogo libros y agendas de autoayuda y coaching para el público adulto. Este tipo de publicaciones goza de mayor prestigio comercial que estético, es decir, su valoración está dada por la cantidad de libros vendidos. No obstante, no son reconocidos como productos con legitimación cultural y literaria por parte de los sectores más especializados del campo: no son reseñados en revistas de crítica literaria ni incluidos en los programas de la carrera de Letras, tampoco reciben premios de academias literarias. Esto hace que su posición en el campo esté legitimada por el mercado, pero no por la crítica o los ámbitos académicos.

El catálogo de $\mathbf{V} \& \mathbf{R}$ Editoras está formado por varias colecciones: Jóvenes Lectores (romance, romance paranormal, fantasy, thriller/misterio, realista, ciencia ficción, distopía, realismo mágico, drama, autobiografía, New Adult), Preadolescentes (ficción: fantasy, aventuras, ChickLit; no ficción: para chicos aventureros; solo para chicas; 1.000 datos de...; libros de villanos y mentiras; libros interactivos), libros infantiles, inspiración (libros de autoayuda y coaching), libros regalos (libros pensados para 
"Te extraño, te olvido, te amo de nuevo". La construcción del amor en la literatura juvenil argentina

regalar en el día del padre, de la madre, del amigo), libros para colorear, cocina y agendas.

Con respecto a la posición de la autora, Georgina Dritsos, se puede decir que estos son sus primeros y hasta el momento, dos libros editados que le permitieron ser reconocida como escritora. Antes de publicarlos, se especializaba en temáticas juveniles y femeninas, siendo redactora en medios gráficos como Clarín y las revistas para jóvenes LunaTeen y Txt, así como también en las editoriales Atlántida, Perfil y Capital Intelectual. Se desempeñó, además, como subeditora de la revista Luna Teen y actualmente es jefa de prensa de $\mathbf{V} \& \mathbf{R}$ Editoras. Su inserción en el campo literario está posibilitada, entonces, por su trabajo en la editorial y posteriormente, por su condición de escritora. Asimismo, su trayectoria como periodista y su participación en editoriales y revistas juveniles, recorrido que compartía con Cristina Alemany, directora editorial de V\&R en el momento de publicarse los libros, facilitó su posicionamiento.

Finalmente, respecto de las condiciones de producción de las que participó, es posible decir que las publicaciones de estos libros forman parte de un mercado ya constituido, que cuenta con algunas lógicas propias: la oferta de escribir viene organizada por parte de la editorial, a la vez que la fecha de su publicación es pautada con anterioridad. En este sentido, la autora tiene que adaptarse a unas condiciones ya dadas. En el siguiente fragmento, extraído de una comunicación personal con la autora (no publicada, realizada el 4 de diciembre de 2016) da cuenta de que la escritura y edición de su primer libro estuvo sujeta a los plazos de la editorial, permitiendo ver la íntima relación entre producción literaria y mercado:

Realicé toda la producción previa que requiere un libro, pero hacía todo con mucha tranquilidad, sin apurarme. Ya había empezado a escribir pero me lo tomé sin apuro, hasta que un día en la editorial me dijeron: 'está confirmado que tu libro está en el plan de publicaciones para salir en tal fecha, ¿cómo vas con la escritura, vas a llegar a tiempo?'. Ahí recién caí en la cuenta de que iba a haber un libro, que sí lo iban a publicar y que iba a tener que acelerar el proceso. Por suerte, pude terminar de organizar la información, seleccionar los temas, los capítulos y terminar de escribir y de redondear todo como para entregar a tiempo el texto final (entrevista realizada a la autora en el marco de esta investigación en diciembre de $2016^{3}$ ).

\section{LA REFRACCIÓN DEL MUNDO SOCIAL: ¿QUÉ DICEN ESTOS LIBROS RESPECTO DEL AMOR?}

S.O.S. Tengo mi primera cita se publicó en el 2014 y forma parte de la línea No Ficción - Solo para chicas de la colección "Preadolescentes" que tiene la editorial. Es una guía escrita para mujeres de ese rango etario que van a tener su primera cita con un varón. Para ello, la autora da consejos de cómo prepararse y comportarse en la cita. Además, ofrece tests para saber si la lectora está preparada y si el chico está interesado en ella. Finalmente, recomienda una lista de películas norteamericanas, de género comedia romántica, para que las lectoras puedan seguir profundizando sus conocimientos al respecto. Según la autora en la entrevista realizada, "trata sobre todos los temas que hacen a ese momento de la vida cuando te empiezan a gustar los chicos y no tenés ni idea de cómo manejarte, de qué hacer, qué decir en una cita, qué no decir".

Por su parte, Diario de una ruptura se publicó en el 2016 y es clasificado dentro de la línea "Ficción - ChickLit" también de la colección "Preadolescentes". Este libro está dividido en dos partes: la primera es una breve ficción acerca de Brenda, una chica de dieciocho años cuyo novio cortó la relación por medio de un mensaje de texto. Se narra, a modo de diario íntimo, qué hace el personaje en esa situación de ruptura junto con sus amigos y familiares. La segunda parte es una guía de qué hacer y qué no durante el proceso de una ruptura. Se dan consejos y recomiendan películas y canciones para atravesar ese momento. La idea de este libro -según contó la autora en la entrevista- "surgió al terminar el primero. Acordamos con la editorial que escribiría sobre las rupturas amorosas, sobre qué le pasa a una persona cuando corta una relación, cómo se siente,

3 A partir de aquí, todas las transcripciones que se hagan de su voz remitirán a esta entrevista inédita. 
cómo se puede salir adelante y todo eso que nos sucede cuando nos rompen el corazón".

El género "ChickLit" es aplicado a la literatura escrita por y para mujeres que narra, en un tono personal y humorístico, la vida cotidiana de la mujer, abordando temas como el amor (citas, relaciones, matrimonio) la amistad, el trabajo (compañeros, expectativas), representaciones de la belleza (peso, depilación, vestimenta). La denominación surgió para clasificar como "infantiles" y "banales" las columnas que publicaban las mujeres en diarios y revistas, pero se convirtió en un modo particular de referenciar productos de la industria cultural (columnas periodísticas, novelas, películas, series), sobre todo a partir del éxito de "Sex \&The City" y de "El diario de Bridget Jones", representantes paradigmáticas de este género.

Al detenerse en los capítulos no ficcionales, se puede encontrar una continuidad entre las narrativas de cierto tipo de revistas juveniles y estos libros, que se facilita por el catálogo de la editorial y la trayectoria de la autora, pero también por el modo en que están narrados y los recursos que elige: guía de acciones, tests, testimonios en primera persona, recomendaciones musicales, cinematográficas, de vestimenta. Dicho en otras palabras, estos apartados de ambos libros presentan una continuidad con las secciones habituales de las revistas juveniles contemporáneas dirigidas a mujeres, como pueden ser Seventeen, Para Teens, TKM, Luna Teen, Txt, de las que su autora participaba. Dice Dritsos (2014) al respecto:

Provengo del periodismo y mi trabajo anterior fue como subeditora a cargo de una revista para jóvenes y adolescentes donde solía escribir textos sobre el amor, los vínculos, sobre sexualidad, sobre la amistad y otros temas, así que estos libros son un poco una prolongación de toda aquella experiencia previa.

Recuperando los aportes de Mijaíl Bajtín (1982) en torno a los géneros discursivos y la novela en particular, es posible advertir que son formas de la plurivocidad propiamente literaria que convoca a discursos pertenecientes a esferas discursivas distintas y mezclados en un nuevo producto. Esta hibrida- ción - es decir, este "sistema de fusión de lenguajes, literariamente organizado" (Bajtín, 1978, citado en Altamirano y Sarlo, 1993, pp. 68-69)- puede construir lo que Bajtín denominó "géneros intercalados". Estos géneros construyen una conciencia galileana, descentrada, que relativiza los límites entre los géneros (Altamirano y Sarlo, 1993).

Ahora bien, ¿qué es "lo literario" en estos libros? ¿Por qué son considerados literatura si utilizan, en gran parte, el discurso de las revistas juveniles? En primer lugar, podría decirse que se debe a que la incorporación de otros discursos está subordinada a una función estética y es esta la que prima en los libros. Su predominio, entonces, no excluye la actividad simultánea de otras funciones. Si hay tests, instructivos, recomendaciones, es en la medida en que contribuyen a construir una narración acerca de la primera cita o la separación con una pareja, según cada libro. Pero como se mencionó al principio, no se puede definir una obra literaria por la mera descripción de sus elementos, sino que es necesario pensarla en relación. Siguiendo a Williams (1977), "es preciso rechazar 'lo estético' tanto como dimensión abstracta separada o como función abstracta separada. Es preciso separar la 'Estética' en la medida en que se apoya en estas abstracciones. Al mismo tiempo se debe reconocer y, aún más, subrayar las intenciones específicas variables y las respuestas específicas variables que han sido reconocidas como estéticas" (Williams, 1977, citado en Altamirano y Sarlo, 1980, p. 45). En este sentido, su definición viene anudada a lo que la sociedad -O por lo menos un sector especializado de ella- asigne como estético y por lo tanto, literario en un momento dado. ¿Hubieran sido considerados literarios estos libros en otro momento sin la legitimación de los discursos que convoca? Probablemente, no.

Avanzando con el análisis de la puesta en forma de estas obras, se dice que son libros juveniles que tematizan el amor. Toda obra literaria se inserta en un espacio discursivo más amplio, del que se nutre y al que construye. En otras palabras, se puede leer en la literatura un doble juego: a) la incorporación de discursos y saberes contemporáneos, es decir, la refracción del mundo social y b) la constitución de la literatura en tanto fuente de las representaciones de una época (Sapiro, 2016). 
"Te extraño, te olvido, te amo de nuevo". La construcción del amor en la literatura juvenil argentina

En este sentido, se evidencia que estos libros recuperan matrices discursivas respecto del amor que circulan socialmente. Se entiende que una matriz discursiva funciona como un "molde" que da forma a la enunciación. Los elementos que formen parte de la matriz pueden variar según las circunstancias, hacerse foco en unos más que en otros, pero mantiene una estabilidad. Al inscribirse en determinada matriz discursiva, los enunciados tienen rasgos comunes, así como límites que marcan lo que es posible decir y lo que no dentro de ese molde. Siguiendo lo que plantea Arnoux (2008, p. 11), "'matriz discursiva' remite tanto a un espacio de regularidades generador de discursividad como a un molde que permite dar forma discursiva a datos diversos e, incluso funcionar como grilla interpretativa de lo social".

Una de las matrices que se recupera y reconstruye en los libros es la matriz del amor romántico. Este tipo de amor propone a la pareja como la "media naranja" o el "alma gemela", a la que se le debe y exige exclusividad a cambio de un amor único e insustituible que completa al sujeto. En esta línea, se demanda fidelidad y se construye un tipo de subjetividad como "ser para otro" (Fernández, 1993). Así, supone y construye relaciones desiguales entre "lo masculino" y "lo femenino", depositando en el primero mayor autonomía y en el segundo, subordinación a través de una fragilización de su subjetividad. En este marco, "lo masculino" está asociado con la seguridad, la comprensión, la perfección, mientras que "lo femenino", con la inseguridad, la vulnerabilidad y la fragilidad.

S.O.S. Tengo mi primera cita en principio está destinado solo a chicas, desde la clasificación de la editorial y el uso de los pronombres femeninos por parte de la escritora, como si fuesen las mujeres las únicas que necesitaran una ayuda que las organice y les diga qué hacer en situaciones que implican una relación erótico-afectiva. En un cruce de las condiciones de género y edad, las mujeres jóvenes son construidas como inseguras, miedosas, nerviosas y alteradas en tanto piden ayuda -el pedido S.O.S. ya está inscripto en su título- para salvarse. Se puede ver esta construcción en el inicio del libro:
Todavía faltan varias horas para LA cita con ÉL... No hay ninguna prisa. La casa está tranquila, pero en tu interior estás muerta de miedo, nerviosa, alterada. Tu cabeza es un torbellino de pensamientos, de inseguridades. No puedes evitar pensar: ¿y si algo sale mal? ¿Si no la paso bien con él? ¿Y si no me gusta? ¿O si no le gusto y me deja plantada en el medio de la cita? O peor aún: ¿si ni siquiera viene a buscarme? iißSocorroooo!!! (se respeta el subrayado de la publicación). (Dritsos, 2014, p. 3).

En esta línea, también se refuerza la cis-heteronormatividad -el libro describe a una chica que espera encontrarse con "ÉL", en mayúscula, grande, fuertey en paralelo, propone relaciones desiguales entre hombres y mujeres: mientras se sigue asociando la mujer con los sentimientos y las emociones, fragilizando su subjetividad (Fernández, 1993), se reafirman y legitiman algunas prácticas machistas de los hombres.

En uno de los apartados de la publicación se construye una tipología de los chicos - deportistas, mujeriegos, nerds, nenes de mamá, depresivos, histriónicos-y el manipulador es descripto como un hombre que "monta un berrinche por cualquier cosa", "lo que quiere es manejarte", "es celoso y posesivo" y "se quejará en voz alta si no haces las cosas a su manera". La descripción termina diciendo que este tipo de chico "no es apto para chicas con personalidad fuerte" (Dritsos, 2014, p. 20). ¿Acaso sí es apto para las chicas sumisas? Dentro de esta matriz, los celos y los deseos de posesión no están inscriptos en un entramado de relaciones de poder desiguales entre hombres y mujeres, sino como dinámicas propias de una relación erótico-afectiva.

Del mismo modo, en el apartado del libro que reconstruye los momentos previos a la cita, se reproduce un posible diálogo familiar en el que el padre asegura:

"¡Ponte cualquier cosa menos una de tus minifaldas si no, no te dejo salir!" y se agrega más adelante: "¡Ah! Y no te olvides de decirle a ese inútil que soy cinturón negro de karate (papi no hace karate desde los 
18 años, ipero está convencido de que es el mejor karateka del planeta!)". (Dritsos, 2014, p. 7).

Así, mientras que la mujer es vinculada con los sentimientos, el hombre lo es con la fuerza, la violencia y la autoridad. Esta matriz, entonces, construye y sostiene un orden social jerarquizado, "un orden, asimismo, heterosexual, que implica no solo privilegiar una forma de deseo frente a otras posibles, sino una forma de entender las relaciones entre lo masculino y lo femenino absolutamente dicotómica y complementarista" (Esteban, 2011, p. 48).

Por su parte, esta matriz romántica también está presente en Diario de una ruptura, el segundo libro de Dritsos. La primera parte, como se mencionó, es una breve historia ficcional que -a modo de diario personal- aborda los días posteriores a la ruptura de un noviazgo de un año y medio. La segunda tiene una perspectiva similar al primer libro, es decir, se posiciona como una guía para "curar el corazón roto".

La historia ficcional comienza con el mensaje de texto que le envía Alex (de 24 años, "inteligente, atractivo y candidato perfecto", estudiante de Derecho con el mejor promedio de su año) a Brenda, una chica de 18 años que acaba de terminar la escuela secundaria, no sabe qué va a estudiar y "por primera vez en su vida está enamorada". A partir de aquí se la describe como "intranquila", en un "estado de nervios y ansiedad", con el corazón "latiendo a mil por horas", con "ganas de querer matarlo", "los ojos llenos de lágrimas y la cabeza abombada" y "furiosa". Como la historia es contada desde la perspectiva del personaje femenino, no se hace mención a los sentimientos y las emociones de Alex. Solo se incluyen las palabras que envió por mensaje de texto: "Lo nuestro terminó, Bren. Ya no quiero estar contigo. $Y$ no llores, no es el fin del mundo. Suerte en tu vida. Adiós". (Dritsos, 2016, p.8).

La construcción de esta voz narradora, dentro del repertorio de posibles, pone el acento en Brenda, con un estilo de escritura íntima y subjetiva: solamente se sabe lo que va ocurriendo través de la mirada del personaje femenino. Este gesto, a la vez que ubica a una mujer como protagonista, privilegia la narración de sus sentimientos y obtura los sentimientos en el personaje masculino. Podría decirse, hasta aquí, que las emociones siguen siendo territorio exclusivo de las mujeres, mientras que a los hombres se les quita la capacidad de sentir. Su ámbito es el de las palabras, el de la racionalidad. El personaje masculino supone a priori que Brenda, en tanto mujer, va a llorar al leer el mensaje de texto. Él - en cambio- puede decir con tranquilidad que "no es el fin del mundo".

Al mismo tiempo, es posible afirmar que se sostiene la construcción de una relación desigual y asimétrica en donde las mujeres siempre representan la subalternidad: Brenda es más chica y más inexperta mientras que Alex es "inteligente y le espera un futuro brillante", "muuuuy bonito, tanto que ahora duele recordarlo", "gran deportista y tiene el cuerpo marcado", "buen hijo, buen hermano, buen amigo", es decir: "perfecto". (Dritsos, 2016, p. 41). Se construye, de este modo, una hipermasculinidad más poderosa que encanta a las mujeres y las posiciona en un lugar dominado, tanto que son siempre ellos los que toman la decisión de terminar con la relación, de "dejar" lo que ya tuvieron por otra mujer.

Ahora bien, entendiendo que los productos culturales pueden ser heterogéneos y complejos, esta matriz no es la única que emerge en los libros. Es decir, la narración oscila entre una matriz de amor romántico -tal como se ha analizado hasta aquíy una matriz que se llamará de amor contingente. Estas matrices discursivas conviven entre sí, no sin contradicciones, en los libros.

En la matriz del amor contingente se revisan algunas de las premisas del amor romántico y se plantean formas diferentes del devenir de la relación eróticoafectiva. En principio, a diferencia de la primera matriz, que supone la pasión eterna en la pareja o por lo menos, una perdurabilidad "hasta que la muerte nos separe", las relaciones erótico-afectivas pueden tener una duración finita. Se rompe, de este modo, con la premisa de la predestinación de los amantes, así como su duración para toda la vida. 
"Te extraño, te olvido, te amo de nuevo". La construcción del amor en la literatura juvenil argentina

Yendo a los libros, en S.O.S. Tengo mi primera cita, uno de los apartados propone las derivas que puede tener ese primer encuentro: puede ser que no se conozca al amor de la vida, puede ser que no implique el inicio de una relación, puede incluso ser insignificante. Dice la autora:

Toda cita es un "riesgo", en el sentido de que puede salir bien, mal o ser simplemente un momento más que pasa sin pena ni gloria en tu vida. Si una no se quiere "arriesgar", es decir, exponerse, más vale quedarse en casa, en donde no te "arriesgarás" a que alguien te deje plantada, o a pasar un rato con un chico que, quizás, finalmente no te interese, entre otras situaciones posibles... pero tampoco vivirás la vida como se supone que hay que hacerlo: viviéndola a pleno. Como dice el dicho: "el que no arriesga no gana" (Dritsos, 2014, p. 13).

Asimismo, esta matriz no sostiene la construcción de un orden desigual y desjerarquizado entre hombres y mujeres: los hombres no son, necesariamente, perfectos y las mujeres no son, necesariamente, sensibles. En cambio, se habilita una constelación de prácticas y representaciones de lo femenino que, en la matriz romántica, estaban obturadas.

En Diario de una ruptura, a la semana de terminar la relación con su novio, Brenda viaja a la casa de su abuela Dora, que está ubicada en un pueblo costero. Su abuela, lejos de responder a las representaciones hegemónicas en torno a la adultez, es "joven y cool": "toca la guitarra, algo de piano y baila música electrónica. Además, practica yoga, es una gran lectora, una excelente cocinera y aparenta unos diez años menos de los que tiene", usa Facebook y Skype, tiene tatuajes, está casada por tercera vez y se la muestra con una vida sexual activa (Dritsos, 2016, p. 24). De este modo, se construye un tipo de feminidad adulta que no se corresponde con las actividades esperables para su género y su generación: cuidado de la familia y el hogar, tareas manuales, consumos culturales de otra época, un cuerpo inmóvil, el placer y el goce obturados. En cambio, hay una construcción juvenilizada, como una nueva expectativa para el ciclo vital de las mu- jeres, que la convierte en "una mujer fuerte, decidida; una muñeca brava" (Dritsos, 2016, p.44).

En esta misma línea, en el devenir de la historia se presenta la ruptura como un momento doloroso para Brenda, pero también como una oportunidad para salir "fortalecida" y descubrirse a sí misma. En este argumento, todo momento, por más angustiante que pueda resultar, es visto como una oportunidad de aprendizaje y crecimiento. En el caso de Brenda, es una oportunidad para darse cuenta de que Alex no era "perfecto" como lo creía, sino celoso y posesivo. Más importante aún fue lograr prestarle atención a aspectos de su vida que antes no hacía o no se animaba, por ejemplo, observar su cuerpo y descubrir que está conforme con él. Al final del libro, este personaje reflexiona:

Lo que sí tengo en claro es que no voy a descuidar otros terrenos de mi vida que son importantes para mí, tanto o más que el amor. Me refiero a mi futuro profesional. Apenas pueda voy a inscribirme en la carrera de Letras que es mi verdadera vocación: la literatura y, a futuro, mi deseo es ser escritora. ¡Ah! También voy a comenzar a tomar clases de danzas árabes con Lara [su amiga], algo que quiere compartir conmigo desde hace tiempo y yo, por miedos e inseguridades, siempre me negué a hacer (Dritsos, 2016, p. 50).

Ahora, en el centro de su vida están sus deseos y proyectos, y el amor es solo una parte. De este modo, ya no se enfatiza el amor por delante de otras facetas humanas ni se busca la felicidad y realización personal en una relación erótico-afectiva, como propone el amor romántico (Esteban, 2011). El consumo musical, de películas, estar con amigos y familiares, y escribir diarios personales le permitieron reflexionar sobre sí misma y sus relaciones, autorrepresentarse y desplegar algunas prácticas para modificar sus emociones y transitar de un estado anímico indeseado a otro en el que "no está tan mal". En este punto, también podría decirse que el amor no aparece como la única preocupación femenina, pero sí emergen otros mandatos de género actuales: la realización profesional y la independencia económica. 
Anudada a esta matriz del amor contingente, es posible encontrar la incorporación en la narración de un discurso de autoayuda en tanto exaltación de la subjetividad y constitución de una guía práctica para superar obstáculos. Aunque estos libros no asuman esa clasificación genérica, se inscriben en un discurso que se extiende más allá de sus publicaciones específicas.

El discurso de autoayuda se constituye en un lugar que orienta la acción de los sujetos en momentos críticos de sus trayectorias y los ayuda a resolver los malestares subjetivos que devienen de ellos. Si bien -como sostiene Vanina Papalini (2015, p. 25)- estas narrativas pueden funcionar como un "dispositivo adaptativo" que "renueva la adhesión a la sociedad instituida" y ubica al yo en el centro de los problemas y las soluciones, también puede ser un apoyo y un acompañamiento en determinados momentos de la vida y producir un posible empoderamiento personal.

Como plantea Illouz (2010):

(...) tanto las novelas como la literatura de autoayuda, cada una de diferente manera, ofrecen escenarios a través de los cuales los actores pueden ensayar cognitivamente su experiencia emocional y reflejarse en las transacciones y en las expresiones emocionales de otros. Al hacerlo, los actores otorgan sentido a sus propios sentimientos (y a los de otros), prescriben sutilmente reglas para tratar con las emociones y proporcionan un vocabulario y un método de introspección (p. 33).

Se puede ver, entonces, que los géneros literarios -como todo género discursivo- no son individuales ni singulares, obra de un sujeto aislado, así como tampoco son estructuras homogéneas y estáticas. Son más bien formas dinámicas, ligadas a las prácticas sociales y sujetas a las transformaciones que devienen en la práctica (Di Stéfano, 2015). Esto habilita que la definición de un género varíe en el tiempo, incorpore elementos de otras esferas discursivas y se tensionen sus propios límites.

\section{CIRCULACIÓN, APROPIACIONES Y LECTURAS}

Esta tercera dimensión de análisis -que busca indagar en la circulación y la recepción de las obras literarias- parte de sostener que el sentido de ellas no se reduce a las intenciones de su autor, sino que se completa en las apropiaciones que de ellas hagan los lectores. Como la recepción es un proceso que desborda el marco de la producción de la obra en el tiempo y el espacio -porque hay reediciones, traducciones, relecturas-, es necesario contemplar el contexto socio-histórico en el que se inscribe el estudio de las apropiaciones.

A su vez, no pueden negarse las mediaciones que intervienen en este proceso: edición (inscripción en un catálogo, redacción de la contracubierta, diseño), espacios de circulación (editoriales, librerías, ferias del libro), clasificaciones que realiza la crítica literaria y la prensa, la difusión y consagración (volumen de ventas, premios, consagración institucional). En este sentido, las obras literarias son mediatizadas por las interpretaciones por parte de agentes individuales o institucionales, profesionales como críticos, revistas, jurados, academias o lectores fuera del campo literario, pero con incidencia en el espacio público, como el periodismo.

V\&R Editoras, como se mencionó, pertenece al Sector Editorial Comercial y su circulación también está dada por espacios comerciales hegemónicos: en las cadenas de librerías más grandes de Argentina y las principales ferias del libro del país: la Feria Internacional del Libro de Buenos Aires y la Feria del Libro Infantil y Juvenil, en donde cuenta con stand propio y la posibilidad de organizar actividades todos los días. También tiene sedes en México y Brasil, y distribuye sus libros en América Latina y Estados Unidos. Para los fines de este texto, se recortará el análisis a la recepción que tuvieron ambos libros en Argentina.

Entendiendo que la apropiación de las tecnologías digitales ha habilitado prácticas que trascienden la lectura individual y silenciosa de las obras literarias, en tanto permite que se creen clubes de lectura en plataformas como Lectorati y Goodreads, se publiquen reescrituras -conocidas como Fan Fictionsque pueden luego convertirse en libros (como es el caso de 50 Sombras de Grey, de E.L. James), se 
"Te extraño, te olvido, te amo de nuevo". La construcción del amor en la literatura juvenil argentina

abran canales de YouTube y cuentas en blogs dedicados a la reseña de ellas, lo que da lugar a un nuevo modelo de lector y de crítico (los booktubers y los bookbloggers), es que se puede afirmar que las formas de recepción de la literatura se constituyen en la interface entre lo online y lo offline.

Esto exige -entonces- construir herramientas metodológicas que permitan acercarse a estas prácticas. La etnografía virtual, en esta línea, invita a reconsiderar el carácter de la etnografía ligada a unas coordenadas espaciotemporales concretas, porque las "nuevas tecnologías de la interacción" facilitan que las relaciones se desplacen o se sostengan en diferentes espacios y temporalidades. Por lo tanto, propone pensar en "interacciones mediadas" (Hine, 2004, p. 81) que hacen de la etnografía un método más fluido, dinámico y móvil. Precisamente, el "reto" de la etnografía virtual consiste en "examinar cómo se configuran los límites y las conexiones, especialmente, entre lo 'virtual' y lo 'real'" (Hine 2004, p. 81).

En este marco, se construyó un corpus heterogéneo de materialidades para acercarse a la recepción de los dos libros que se han estado analizando. En primera instancia, la literatura juvenil contemporánea de circulación masiva no es reconocida como una literatura válida de ser reseñada y/o analizada por la crítica y las revistas literarias. Estos libros, en tanto inscriptos en esa producción, no han circulado por esos lugares especializados del campo literario. No obstante, no son estos los únicos espacios por donde un libro puede ser mediatizado. Por ese motivo, este análisis incluye los medios de comunicación, los blogs y canales de YouTube que administran jóvenes lectores, los comentarios que en ellos dejan sus seguidores y los comentarios en la plataforma Goodreads.

S.O.S. Tengo mi primera cita fue reseñado en dos medios de comunicación diferentes entre sí. Uno es la agencia de noticias estatal TELAM y el otro la revista juvenil TKM. En el primero se publica una entrevista a la autora en la sección "Cultura", en donde se destaca el lugar en el campo que construye para sí misma y la publicación al manifestar un vacío editorial en el tratamiento de temas para preadolescentes y adolescentes: "Veo desinformación y hay pocos libros o publicaciones que los guíen en esta etapa de tantos cambios, donde dan sus primeros pasos en el amor" (Télam, 2014, s/p). En este sentido, ubica su libro en un lugar del campo que pareciera no estar explorado y lo plantea como una novedad. Sopesando esta construcción de la autora, quien escribe la nota (no está firmada), asegura que es un libro "al mejor estilo revista femenina", encontrando una continuidad de este tipo de literatura con otros discursos (tal como se explicó en el apartado anterior).

Por su parte, la revista TKM recomienda la lectura de este libro en su sección "Libros y Cómics" para que la lectora se "relaje, divierta y aprenda cómo lidiar con la situación" (TKM, 2016, s/p). Esta recomendación reproduce el resumen que el libro tiene en su contratapa e invita a leerlo en la plataforma Leamos, de suscripción mensual paga para descargar e-books. Se aprecia, aquí, que no circular por los espacios propiamente literarios le posibilita inscribirse en aquellos otros en donde transitan sus potenciales lectores. Diario de una ruptura -en cambio- no tuvo reseñas o comentarios en medios de comunicación tradicionales, sino que todas sus referencias están en otros espacios de circulación más diversos.

A su vez, en cuanto a las reseñas y comentarios que realizaron los lectores en un conjunto de plataformas digitales: blogs, YouTube y Goodreads, estos géneros discursivos no pueden compararse con una reseña en una revista literaria, porque su estilo y composición son diferentes, tienen un registro informal y coloquial. Tampoco con las reseñas o recomendaciones de los medios de comunicación, ya que estas nuevas narraciones se escriben/dicen en primera persona, construyendo una vOz más subjetiva e íntima. Así, no solo narran el contenido del libro, sino también qué les sucedió cuando lo leyeron.

Por otro lado, un aspecto que se tematiza en estas textualidades es la edición: cuando el libro está editado en papel, se comenta su diseño, tamaño y materiales como una dimensión más de análisis. Por último, es importante mencionar que el mayor porcentaje de jóvenes que reseñaron estos libros los obtuvieron como obsequio por parte de la editorial 
o la escritora y en algunos casos, se destaca la relación que tenían con ella por su trabajo en la editorial. Se aprecia, entonces, una búsqueda e interés particular para que los libros circulen por estos nuevos espacios de legitimación entre jóvenes: ya no son los padres, los profesores, los bibliotecarios quienes recomiendan lecturas, sino sus propios pares.

Reconstruyendo los sentidos y las sensaciones (Littau, 2008), es decir, atendiendo no solo a su comprensión, sino también a lo que como lectores sintieron al leer los libros, se encontraron tres maneras de apropiarse de S.O.S. Tengo mi primera cita y Diario de una ruptura. La primera está vinculada con el entrenamiento y la fluidez en la lectura. Ambos libros tienen entre 80 y 100 páginas, y se destaca que la escritura amena y fluida posibilita que puedan leerse en pocas horas e-incluso- de manera colectiva, con amigos.

Se ve -asimismo- como una opción "corta" y "liviana" para leer entre otras lecturas más largas y densas o para salir de un "bloqueo lector". Esta sensación puede -en algunos casos- derivar en que se consideren estos libros como "básicos", "muy breves" o "sin desarrollo de lo que le pasa a los personajes". Respecto del primero, se coincide en decir que es un libro para "divertirse", "distraerse un rato" y "reírse" (Comentarios de lectores publicados en la plataforma Goodreads. Extraído de: https: //www. goodreads.com/book/show/22918110-s-o-s-tengomi-primera-cita, última fecha de consulta: 9 de febrero de 2018).

Del segundo que, como no recurre a la melancolía para hablar de la ruptura de una relación eróticoamorosa, es "entretenido" y sirve para "verle el lado bueno a las cosas" e incluso poder reírse de algunas situaciones vividas por los personajes, sobre todo la relación que tiene Brenda con su abuela. (Comentarios de lectores publicados en la plataforma Goodreads. Extraído de: https: //www.goodreads. com/book/show/28948427-diario-de-una-ruptura, última fecha de consulta: 9 de febrero de 2018).

En palabras de los lectores:

La verdad que [S.O.S Tengo mi primera cita] es un libro sumamente divertido, me he reí- do mucho con las experiencias y anécdotas que nos cuenta la autora.

Clara (blog Fantaseando con libros, extraído de: http: //fantastacioconlibros.blogspot. com.ar/2014/09/resena-sos-tengo-mi-primera-cita.html última fecha de consulta: 9 de febrero de 2018).

Diario de una Ruptura es una lectura breve y dinámica que seguramente te será de gran ayuda para superar un corazón roto o una entretenida lectura para pasar un momento agradable con una historia reflexiva y divertida. Ideal para regalar y regalarse (Stefany, Blog Mi reflejo en el papel, extraído de: http: //fantastacioconlibros.blogspot.com. ar/2014/09/resena-sos-tengo-mi-primeracita.html, última fecha de consulta: 9 de febrero de 2018).

La segunda se refiere a la identificación que sintieron cuando leían, porque se acordaron de experiencias vividas en primeras citas o estaban viviendo en el momento de la lectura una ruptura y leyeron el libro como una guía. Este tipo de apropiación es una de las más estudiadas, en tanto la relación de la lectura con algunos grupos sociales como los sectores populares, las mujeres y los jóvenes, y ha sido nombrada en términos de identificación en estudios anteriores (Viala y Molinié, 1993 y Sapiro, 2011 en Sapiro, 2016). En este contexto, es posible decir que se brinda un marco interpretativo del funcionamiento del mundo social, porque se vincula la experiencia de vida con representaciones sociales interiorizadas por el lector (Sapiro, 2016):

Todas las situaciones que se plantean, desde la previa a la cita, hasta los pensamientos que supuestamente cruzan por nuestra mente, son tal cual a la realidad!!! al menos yo me sentí súper identificada y justamente por eso no podía evitar las risitas que se me escapaban de vez en cuando (Carla, blog Mi mundo está en tus páginas, extraído de: http://mimundoestaentuspaginas.blogspot. com.ar/2014/09/opinion-de-sos-tengo-miprimera-cita.html ${ }_{\alpha}$ última fecha de consulta: 9 de febrero de 2018). 
"Te extraño, te olvido, te amo de nuevo". La construcción del amor en la literatura juvenil argentina

Creo que es un precioso libro [Diario de una ruptura] que cualquier persona en cualquier momento de su vida lo puede leer y sentirse identificado. Me hubiera gustado haber leído algo así cuando me rompieron el corazón o cuando yo misma me lo rompí. Siento que en mi vida, lo voy a releer varias veces (Belén, Goodreads, extraído de: https://www.goodreads.com/ book/show/28948427-diario-de-una-ruptura, última fecha de consulta: 9 de febrero de 2018).

La última forma de apropiación de este libro se relaciona con su función didáctica, es decir, su condición de "dejar enseñanzas" y la posibilidad que se tiene de "reflexionar" y "aprender" no solo qué hacer en una primera cita o cómo superar la ruptura de una relación erótico-amorosa, sino también a valorarse a sí mismo. En este sentido, se asocia la lectura con su dimensión placentera, como en las anteriores apropiaciones y también con su posibilidad de aprendizaje y acompañamiento en determinados momentos de la trayectoria de vida. Incluso ese aprendizaje puede extenderse más allá de los adolescentes a quienes están dirigidos los textos y pueden también sus madres "aprender" cómo se vive la adolescencia y saber qué le pasa a sus hijos.

[S.O.S Tengo mi primera cita] Es un libro que tienen que leer madres e hijas; una para aprender de cómo se dan en actualidad las cosas, otra para comprender que no todo tiene que ser como lo dicen las normas implícitas de esa edad. Sirve para actualizarte, para aprender iy para divertirte! (Florencia, blog Plausible, extraído de: http: //xylobrytes.blogspot.com.ar/2014/10/fotoresena-sos-tengo-mi-primera-cita.html, última fecha de consulta: 9 de febrero de 2018).

Este libro me gustó mucho ya que me encontraba pasando por una ruptura, y a veces me disponía a hacer lo que el libro decía de hecho la lista de canciones y de películas fue la mejor parte, Luis, Goodreads, extraído de: https://www.goodreads.com/book/ show/28948427-diario-de-una-ruptura, última fecha de consulta: 9 de febrero de 2018).

Ideal no solo para los que acaban de terminar una relación de pareja. Cualquiera sea la relación que se te acaba de terminar (de pareja, de amigos, creo que incluso sirve si te despidieron de tu trabajo) este libro es ideal para sobrellevar el tiempo de 'duelo' (Natalia, Goodreads, extraído de: https: //www. goodreads.com/book/show/28948427-diario-de-una-ruptura, última fecha de consulta: 9 de febrero de 2018).

\section{A MODO DE CIERRE}

Este trabajo partió de suspender el juicio estético en torno a los dos libros seleccionados para centrarse en un juicio analítico. En este marco, los libros se abordaron en tanto hechos sociales inscriptos en un campo específico y aún más en un contexto socio-histórico que los hace posibles. Asimismo, se afirma que el análisis de objetos literarios debe involucrar perspectivas que trasciendan la indagación textualista o biográfica, porque se entiende que la literatura no es una práctica individual o aislada, así como tampoco los autores son totalmente libres e innovadores. Hay -en cambio- una regulación de lo dicho: hay condiciones de posibilidad que habilitan lo que se dice y lo que no se dice. Para ello, tal como se hizo a lo largo del trabajo, es necesario reconocer las mediaciones presentes entre los libros y el entramado social. Esto significa tener en cuenta las condiciones sociales de su producción, la puesta en forma de las obras y la circulación y apropiación que de ellas se hacen.

Considerando estas dimensiones es posible sostener -entonces- que los géneros literarios son contextualmente variables y no pueden pensarse por fuera de sus condiciones de posibilidad. ¿La muerte en Venecia, de Thomas Mann, que construye a un escritor alemán enamorado de un joven polaco, podría haber sido considerada una novela romántica al momento de su publicación? ¿Romeo y Julieta, de William Shakespeare, convertida en un hito de la literatura romántica, hubiera sido clasificada dentro de ese género si los personajes, en lugar de suicidarse, hubiesen aceptado no estar juntos? ¿El discurso 
de la autoayuda como parte del género romántico hubiese sido aceptado en tiempos donde la narrativa hegemónica construía un "nosotros" antes que un "yo"?

Ligado a esta inquietud, la emergencia de la matriz del amor contingente y su conflictiva copresencia con la matriz del amor romántico en las novelas que tematizan el amor se inscribe en un contexto que hace posible su enunciación. Es decir, en unas coordenadas históricas donde existen leyes que reconocen los derechos de las mujeres, así como leyes de matrimonio igualitario e identidad de género, crean un clima de mayor respeto y reconocimiento a la diversidad sexual y de género.

Siguiendo lo que propone Illouz (2016 [2011], p. 15), acercarse a las narrativas del amor permite acercarse, a su vez, a "los modos de institucionalización del yo y la identidad en la época moderna". En otras palabras, es un microcosmos privilegiado para entender los procesos del tiempo actual. Asimismo, el sufrimiento amoroso habla de la "desregulación del mercado matrimonial, las transformaciones en la arquitectura de la elección de pareja [y] la importancia del capital del amor en la construcción social de un sentido de valor propio" (Illouz, (2016 [2011], p. 29). Analizar, entonces, objetos literarios desde esta perspectiva ayuda a hacerse preguntas que desbordan los límites del texto, observar el estado del campo y acercarse a las representaciones del entramado social de una época.

\section{REFERENCIAS BIBLIOGRÁFICAS}

Altamirano, C. y Sarlo, B. (1993 [1983]). Literatura/ Sociedad. Buenos Aires: Edicial.

Arnoux, E. (2006). Análisis del discurso: tres modos de abordar materiales de archivo. Buenos Aires: Santiago Arcos.

Arnoux, E. (2008). La inscripción en los relatos modernos en la matriz latinoamericanista. En: El discurso latinoamericanista de Hugo Chávez. Buenos Aires: Biblos.

Badiou, A. (2011). Elogio del amor. Madrid: Esfera de los Libros.
Bajtín, M. (1982). El problema de los géneros discursivos. En: Estética de la creación verbal. México: Siglo XXI.

Bourdieu, P. (2015 [1992]). Las reglas del arte. Génesis y estructura del campo literario. Barcelona: Anagrama.

Cámara Argentina del Libro. (2017). Informe de Producción del Libro Argentino Primer semestre de 2017 [en línea]. Recuperado de https:// issuu.com/camaradellibro/docs/informe_de_ producci_n_del_libro_ar_14f17973019e20.

Cámara Argentina del Libro. (2017). Informe de Producción del Libro Argentino 2016 [en línea]. Recuperado de https://issuu.com/camaradellibro/ docs/informe_de_producci_n_anual_2016_v.

Cámara Argentina del Libro. (2016). Informe de Producción del Libro Argentino 2015 - Resumen Ejecutivo. [en línea]. Recuperado de https:// issuu.com/camaradellibro/docs/2016.03.28_informe_de_producci_n_a.

Cámara Argentina de Publicaciones. (2015). Libro blanco de la industria editorial argentina. Informe de datos estadísticos. [en línea]. Recuperado de http://www.publicaciones.org.ar/Libro $\% 20$ blanco.pdf.

Di Stefano, M. (2015). La noción de discurso y de comunidad discursiva. En: Anarquismo de la Argentina. Una comunidad discursiva. Buenos Aires: Cabiria.

Dritsos, G. (2014). S.O.S. Tengo mi primera cita. Buenos Aires: V\&R Editoras.

Dritsos, G. (2016). Diario de una ruptura. Buenos Aires: V\&R Editoras.

Esteban, M. L. (2011). Crítica del pensamiento amoroso. Barcelona: Belaterra.

Fernández, A. M. (1993). La mujer de la ilusión. Pactos y contratos entre hombres y mujeres. Buenos Aires: Paidós.

Giddens, A. (1998 [1992]). La transformación de la intimidad. Sexualidad, amor y erotismo en las 
"Te extraño, te olvido, te amo de nuevo". La construcción del amor en la literatura juvenil argentina

sociedades modernas. Madrid: Ediciones Cátedra.

Hine, C. (2004). Etnografía virtual. Barcelona: Editorial UOC.

Illouz, E. (2016 [2011]). Por qué duele el amor. Una explicación sociológica. Buenos Aires: Katz Editores y Capital Intelectual.

Illouz, E. (2014). Erotismo de autoayuda. Cincuenta sombras de Grey y el nuevo orden romántico. Buenos Aires: Katz Editores y Capital Intelectual.

Illouz, E. (2010). La salvación del alma moderna. Terapia, emociones y la cultura de la autoayuda. Buenos Aires: Katz Editores y Capital Intelectual.

Littau, K. (2008). Teorías de la lectura. Libros, cuerpos y bibliomanía. Buenos Aires: Manantial.

Papalini, V. (2015). Garantías de felicidad. Estudios sobre los libros de autoayuda. Buenos Aires: Adriana Hidalgo Editora.

Sapiro, G. (2016). La sociología de la literatura. Buenos Aires: FCE.

Telam. (2014). Una guía para chicas sobre la primera cita con varones [en línea]. Recuperado de http://www.telam.com.ar/notas/201409/78061una-guia-para-chicas-sobre-la-primeracita-con-varones.html, última fecha de consulta: 9 de febrero de 2018.

TKM. (2016). Recomendado: S.O.S. ¡Tengo mi primera cita! [en línea] Recuperado de https://www. mundotkm.com/ar/libros-y-comics/707701/ recomendado-s-o-s-tengo-mi-primera-cita, última fecha de consulta: 9 de febrero de 2018. 